\title{
Toward an "Equal-Footing" Human-Robot Interaction for Fully Autonomous Vehicles
}

\author{
Theocharis Amanatidis, Patrick Langdon, P. John Clarkson \\ Cambridge University Engineering Department, Engineering Design Centre, Trumpington \\ Street \\ Cambridge CB2 1PZ, United Kingdom \\ $\{$ ta323, pml24, pjc10\}@cam.ac.uk
}

\begin{abstract}
Fully autonomous vehicles can be classified as robots. In this paper we propose to approach the development of autonomous vehicle user interfaces from a Human-Robot Interaction perspective, based on two principles. First, different robots require different user interfaces depending on their level of automation. Second, as the level of robot automation increases so should the automation of the interface itself; creating a spectrum ranging from a conventional "masterslave" level interaction to a fully intelligent "equal-footing" level interaction. Two research questions arise: where along the spectrum described above should autonomous vehicle user interfaces be, and what technological advance would have the greatest impact in enabling those interfaces. This paper presents the theoretical foundation of our research at the intersection of three previously unconnected fields: autonomous vehicles, human-robot interaction and affective computing. We then outline an experimental framework for developing a prototype interface based on our findings.
\end{abstract}

Keywords: Autonomous Vehicles · Human-Robot Interaction · Affective Computing

\section{Introduction}

The need for autonomous transportation is thought to arise from a combination of two societal factors [1]. The first is economic: population size of metropolitan areas is expanding, leading to increased congestion and cost of private vehicle ownership. The second is demographic: the median age of citizens in western societies is rising, causing reduced functional ability in an ever larger part of society. It would therefore be beneficial to develop measures to reduce the dependency of transportation on manual operation; one of the sort is the user interface of current automobiles [2]. One possible way to achieve this is through partial but progressively increasing autonomous control, all the way to fully autonomous vehicles [3]. Autonomous vehicles could allow the vehicle to be shared between a larger number of users with minimal inconvenience, collecting customers from and delivering customers to different locations [2]. This would reduce the number of vehicles needed per capita, reducing ownership costs and congestion [1]. Moreover, it will provide customers with reduced capabilities a means of transportation not previously available to them [4]. Yet this shift is dependent on developing an appropriate user interface that would replace traditional automotive controls. 
The Traveller Needs and UK Capability Study identifies two groups of users that would immediately benefit from the shift described above: progressive metropolites (tech-savvy young professionals in urban areas) and dependant passengers (young, elderly or people with impairments who usually don't hold a driver's licence) [2].

Our research goal is thus to develop a user interface for self-driving vehicles. In this paper, we will set the theoretical foundation of our research to achieve this goal using the following three-step process: understand the context of autonomous vehicles, use the principles of human-robot interaction and implement some technological advances of affective computing. Each step is discussed in a section below.

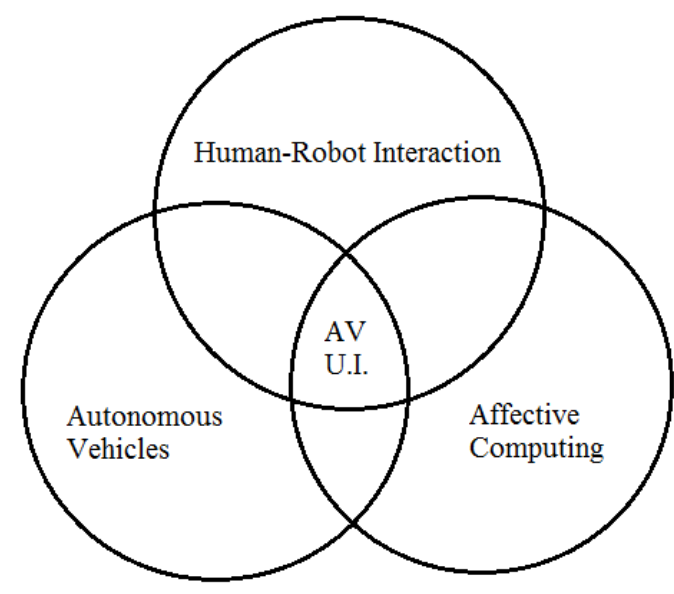

Fig. 1. Visualisation of our investigation for autonomous vehicle (AV) user interface development at the intersection of autonomous vehicle, human-robot interaction and affective computing.

\section{Autonomous Vehicle User Interfaces}

Autonomous vehicle research has progressed rapidly, to the stage that public trials have begun or are ready to begin in the coming months [5]. While extensive research has already been conducted on autonomous vehicle control systems, the development of their user interface is still in its infancy. In order to take advantage of the potential benefits of fully self-driving vehicles, a natural, inclusively designed user interface is needed. This interface should be designed to take into consideration the diverse range of capabilities and needs of different members of society and traveller types [2].

\subsection{Levels of Autonomy in Self-Driving Vehicles}

To begin the process of investigating the design of such interface, it is important to understand the classification of different levels of autonomous vehicles. Each level requires a different type of interaction from the user, as discussed below. For the purposes of this paper the classification of different levels of autonomy in autonomous vehicles 
is based on the SAE J3016 standard [6], summarised in Fig. 1. Fully autonomous vehicles are defined as Level 5 automation on the SAE standard, and could be designed without any traditional manual controls such as a steering wheel or pedals. Vehicles in this highest level of automation do not require any user interaction to operate, even in emergency situations, other than selecting a destination and reporting progress. As such, the user may be out-of-the-loop for the entirety of the journey, allowing for minimal situational awareness and hence for a fully immersive interface if desired [7]. It is hence possible that users of these vehicles will not have to have a driving licence or be otherwise unable to drive due to age, temporary or permanent capability loss. Level 5 vehicles have thus the opportunity to achieve the goal of providing the freedom of the automobile to members of society that were previously excluded by capability [4]. The present paper focus on this level of automation.

Table 1. Summary of SAE J3016 automation levels and their description [6]. The dashed line signifies the transition from a lower to a higher degree of automation between the different levels.

\begin{tabular}{lllll}
\hline Automation Level and Name & $\begin{array}{l}\text { Execution } \\
\text { of Control }\end{array}$ & $\begin{array}{l}\text { Environment } \\
\text { Monitoring }\end{array}$ & $\begin{array}{l}\text { Emergency } \\
\text { Response }\end{array}$ & System Capability \\
\hline 0- No Automation & Human & Human & Human & Some Scenarios \\
1-Driver Assistance & Shared & Human & Human & Some Scenarios \\
\hdashline 2-Partial Automation & System & Human & Human & Some Scenarios \\
3- Conditional Automation & System & System & Human & Some Scenarios \\
4- High Automation & System & System & System & Some Scenarios \\
5- Full Automation & System & System & System & All Scenarios \\
\hline
\end{tabular}

\subsection{Related Work}

A considerable amount of research has been undertaken on the user interface of partially and conditionally autonomous vehicles (Levels 2 and 3 on the SAE scale). The main focus is on designing the handover of control between periods of automated and manual driving - for a summary see [8]. Yet, as described in section 2.1 above, in the context of Level 5 fully autonomous vehicles there will be no handover of control and hence potential designs are free to take a more creative approach to user interface design.

Surprisingly little research has focused on the issues specific to fully autonomous vehicle interfaces. One of the earliest studies was part of the ARGO autonomous vehicle project [9]. While the first prototype was based on a conventional vehicle dashboard, the second used a handheld Personal Digital Assistance computer (PDA) to control the vehicle. This would theoretically enable vehicle control from different seats or being passed on between different users in the car. This approach seems to be copied by the majority of recent projects using the modern equivalents of a PDA: a smartphone or tablet, such in concept cars by Rinspeed [10] or Mercedes [11]. Furthermore, it restricts the number of modalities used to visual, auditory and perhaps haptic, is not adaptable to user's capabilities and requires some level of prior knowledge or training. 


\section{Human-Robot Interaction for Autonomous Vehicles}

Both historically [12] and by definition, fully autonomous vehicles can be classified as robots. For instance, the Cambridge Dictionary defines a robot as "a machine controlled by a computer that is used to perform jobs automatically" [13]. Therefore, we propose to investigate developing a user interface from a human-robot interaction foundation.

\subsection{Spectrum of Autonomy in Human-Robot Interaction}

Thrun argues that human-robot interaction cannot be studied without taking into account the level of autonomy of the robot [14]. This is because the level of autonomy will, along with other factors such as environment, determine the kind of tasks a robot can perform and the expectations humans will have of it. He defines two types of human robot interaction based on the two extremes of robot automation: what he calls indirect and direct interaction. In indirect interaction, as can be found in industrial robots, the user operates the robot as a "master" and the robot executes the command as a "slave". In direct interaction, as could be imagined with an artificial intelligence (AI) agent, the user and robot interact with each other on an "equal-footing". Thus, Thrun proposes a framework for human-robot interaction based on two principles. First, that different robots require different user interfaces depending on their level of automation. Second, as the level of robot automation increases so should the automation of the interface itself. While Thrun argues there are only two distinct types of interaction described above, Yanco argues that there is a continuum of robot autonomy and that the amount of intervention varies [15]. Based on these two assessments we propose there is a spectrum of autonomy in human-robot interaction which reflects the autonomy of the robot. A visual representation of this is shown in Fig. 2 below.

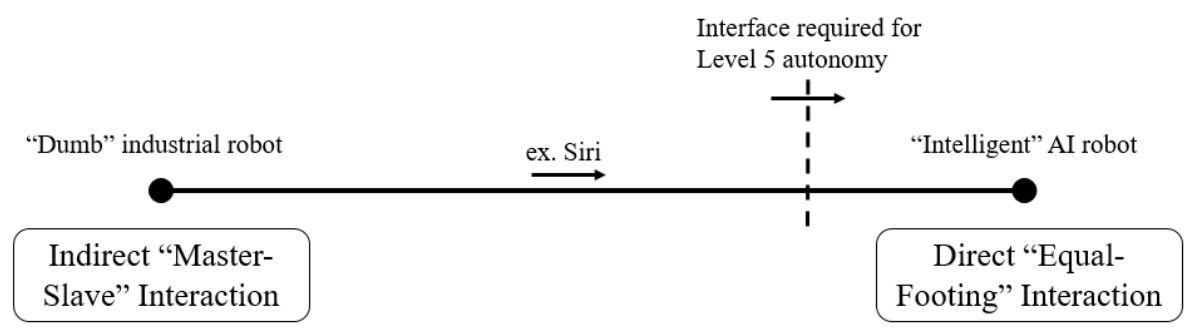

Fig. 2. Proposed spectrum of autonomy in human-robot interaction, based on the work by Thrun [14] and Yanco [15]. We aim to find where the dashed line is located along this spectrum.

\subsection{Autonomous Vehicle Interfaces Based on the Spectrum of Autonomy}

The spectrum of autonomy in human-robot interaction is of importance when trying to understand what kind of interface would be beneficial for an autonomous vehicle user interface. Two research questions arise from its study: where along the spectrum described above should our user interface be? And, what technological advance would have the greatest impact in enabling that interface? 
Some researchers argue that the ultimate goal is to reach the end of the spectrum and an "equal-footing" interaction that would mimic human-human interactions, such as for instance between taxi driver and user. Norman argues that robots should adapt to humans rather than the other way around, and believes humans are much better at interactions at the "equal-footing" versus "master-slave" level [16]. This is reinforced by Stanton and Norman [17], who argue that "the problem is not that automation is too powerful, the problem is that it's not powerful enough" and that feedback and "conversation" is required. On the other hand, Thrun is unsure users would want to interact with robots the same way they do with humans or animals [14]. Finally, Pritchett and Feary warn about using findings based on human-human interaction to human-automation and human-robot interactions [18].

Given the above lack of agreement between researchers we believe that these questions may be answered experimentally, and we propose a plan of how to do so in a later section. Furthermore, the next section will present some possibilities that affective computing can bring to help move towards "equal-footing" interaction in fully autonomous vehicles.

\section{Affective Computing in Autonomous Vehicle User Interfaces}

Picard defines affective computing as: "computing that relates to, arises from or influences emotions" [19]. Based on this definition and her eponymous work, she argues that affective computing is not necessarily creating a machine with emotions but making a machine understand and/or convey emotions is sufficient to qualify as an affective machine. In this section we try to understand the motivation for using affective computing technologies in autonomous vehicle user interfaces and investigate some technological advances that may help develop an affective user interface.

Scheutz argues there are three benefits to affective computing: emotions make agents or robots more believable, recognising emotions is crucial to adapt to user needs and emotions are an integral part of control of complex agents or robots [20]. Similarly, Jaimes and Sebe argue that human-robot interaction that can sense the affective states of humans and accordingly adapt their behaviour are "likely to be perceived as more natural, efficacious and trustworthy" [21]. In the same text Jaimes and Sebe also conduct a thorough literature review of emotion recognition from facial expressions and audio. Their main findings are that these systems can differentiate between a small number of basic emotions relatively accurately but are not context-sensitive and do not analyse emotions on long enough timescales to infer mood or attitude. Finally, they suggest there may be unexplored potential in multi-modal emotion recognition. The two shortcomings discussed and the recommendation on multi-modal recognition are of importance for autonomous vehicle interaction and therefore need to be addressed.

We believe it is important to conclude with the motivation for affective, natural user interfaces in autonomous vehicles. This motivation is fourfold: 1. Improve user experience, 2. Enable customisation/personalisation, 3. Reinforce the brand and 4. Increase inclusivity. We propose a system with facial and voice/tonal emotion recognition as discussed above as input and a virtual agent in the form of pilot or driver as output. Such a system could increase the levels of trust for new users and would be a step 
towards providing a chauffeur in every vehicle. The level of customisation and personalisation would vastly increase compared to existing systems, could adapt depending on situation or scenario and would constantly keep up to date with the latest trends. This system would also reinforce brand identity, be the differentiating factor between brands and be the face of the company not just in the vehicle, but potentially also in advertisements and showrooms. Finally, it would reduce any required training and enable new higher levels of inclusivity by adapting to each user's needs and capabilities.

\section{$5 \quad$ Future Work and Conclusions}

Based on the theoretical background presented in this paper, we plan to answer our research questions experimentally and develop a prototype interface based on our findings. Our process will be to first perform experiments in the low fidelity environment of a driving simulator and progressively increase fidelity. Initial experiments would use Wizard of $\mathrm{Oz}$ techniques for facial emotion recognition and natural language dialog. Experiments of increasing fidelity will use software tools for facial and voice recognition that are publically available in order to determine what technological advances may have the most impact.

In conclusion, this paper presented the theoretical foundation of our research at the intersection of three previously unconnected fields: autonomous vehicles, humanrobot interaction and affective computing. We first discussed the different levels of autonomous vehicles and presented previous work on autonomous vehicle user interfaces within the fully autonomous context. We then discussed two principles of human robot interaction and proposed a spectrum of autonomy in human-robot interaction that we used to elicit our research questions. Finally, we presented our motivation for the adoption of affective computing technologies in autonomous vehicle interfaces and discussed the benefits and shortcoming of some of them. We will use this approach as a useful guide in developing and evaluating user interfaces for fully autonomous vehicles.

\section{References}

1. Mitchell, W.J., Borroni-Bird, C.E., Burns, L.D.: Reinventing the Automobile - Personal Urban Mobility for the $21^{\text {st }}$ Century, The MIT Press: Cambridge (2010)

2. Transportation Systems Catapult Traveller Needs and UK Capability Study, ts.catapult.org.uk/wp-content/uploads/2016/04/Traveller-Needs-Study-1.pdf

3. Flemisch, F. et al: Design of Human Computer Interfaces for Highly Automated Vehicles in the EU-Project HAVEit. UAHCI, vol. 6767, pp. 270-279 (2011)

4. Jeon, M. et al: Towards Life-Long Mobility: Accessible Transportation with Automation. AutoUI Adjunct, pp. 203-208 (2016)

5. UK Autodrive, www.ukautodrive.com

6. SAE J3016 Standard, www.sae.org/misc/pdfs/automated_driving.pdf

7. Endsley, M.R.: Designing for Situation Awareness: An Approach to User-Centered Design. CRC Press: Boca Raton (2016)

8. McCall, R. et al: Towards A Taxonomy of Autonomous Vehicle Handover Situations. AutoUI, pp. 193-200 (2016)

9. Cellario, M.: Human-Centered Intelligent Vehicles: Toward Multimodal Interface Integration." IEEE Intelligent Systems, vol. 16.4, pp. 78-81 (2001) 
10. Rinspeed, http://www.rinspeed.eu/aktuelles.php?aid=17

11. Mercedes, www.mercedes-benz.com/en/mercedes-benz/innovation/research-vehicle-f-015luxury-in-motion/

12. Moravec, H.P. The Stanford Cart and the CMU Rover. IEEE, vol. 71.7, pp. 872-884 (1983)

13. Cambridge Dictionary, dictionary.cambridge.org/dictionary/english/robot

14. Thrun, S.: Toward a Framework for Human-Robot Interaction. HCI, vol.19, pp. 9-24 (2004)

15. Yanco, H.A., Drury, J.L.: A Taxonomy for Human-Robot Interaction. AAAI Fall Symposium on Human-Robot Interaction, pp. 111-119. 2002.

16. Norman, D.A.: The Design of Everyday Things: Revised and Expanded Edition. Basic Books (2013)

17. Stanton, N.A. et al: The Psychology of Driving Automation: A Discussion with Professor Don Norman." IJVD, vol. 45.3, pp. 289-306 (2007)

18. Pritchett, A., Feary, M.: Designing Human-Automation Interaction. In: Boy, G.A. (eds): The Handbook of Human-Machine Interaction: A human-centered design approach. Ashgate: Farnham (2011)

19. Picard, R.: Affective Computing. The MIT Press: Cambridge (1997)

20. Scheutz, M.: Artificial Emotions and Machine Consciousness. In: Frankish, K., Ramsey, W.M. (eds). The Cambridge Handbook of Artificial Intelligence. Cambridge University Press: Cambridge (2014)

21. Jaimes, A., Sebe, N.: Multimodal human-computer interaction: A survey. Computer Vision and Image Understanding, vol. 108 pp. 116-134 (2007) 